\title{
Mortality Rate Associated with Diabetes: Outcomes From a General Practice Level Analysis in England Using the Royal College of General Practitioners (RCGP) Database Indicate Stability Over a 15 Year Period
}

\author{
Adrian Heald (D) - Mike Stedman · Adam Robinson • Mark Davies • \\ Mark Livingston · Ramadan Alshames · Gabriela Moreno • \\ Roger Gadsby · Gerry Rayman • Martin Gibson · Simon de Lusignan • \\ Martin Whyte
}

Received: November 28, 2021 / Accepted: January 28, 2022 / Published online: February 21, 2022

(C) The Author(s) 2022

\section{ABSTRACT}

Introduction: Total population mortality rates have been falling and life expectancy increasing for more than 30 years. Diabetes remains a significant risk factor for premature death. Here we used the Oxford Royal College of General Practitioners Research and Surveillance Centre (RCGP RSC) practices to determine diabetes-related vs non-diabetes-related mortality rates.

Methods: RCGP RSC data were provided on annual patient numbers and deaths, at practice

\section{A. Heald $(\bowtie) \cdot$ M. Gibson}

The School of Medicine and Manchester Academic Health Sciences Centre, Manchester University,

Manchester, UK

e-mail: adrian.heald@manchester.ac.uk

A. Heald · A. Robinson · M. Gibson

Department of Endocrinology and Diabetes, Salford

Royal Hospital, Salford M6 8HD, UK

M. Stedman · M. Davies

Res Consortium, Andover, Hampshire, UK

M. Livingston

Black Country Pathology Services, Walsall Manor

Hospital, Walsall, UK

R. Alshames

Biochemistry Department, Faculty of Dentistry,

Tripoli University, Tripoli, Libya, UK level, for those with and without diabetes across four age groups ( $<50,50-64,65-79, \geq 80$ years) over 15 years. Investment in diabetes control, as measured by the cost of primary care medication, was also taken from GP prescribing data.

Results: We included 527 general practices. Over the period 2004-2019, there was no significant change in life years lost, which varied between 4.6 and 5.1 years over this period. The proportion of all diabetes deaths by age band was significantly higher in the 65-79 years age group for men and women with diabetes than for their non-diabetic counterparts. For the year

\section{G. Moreno}

Marina Nacional 162, Anáhuac Secc, Miguel

Hidalgo, 11320 Mexico City, Mexico

R. Gadsby

Warwick Medical School, University of Warwick, Warwick, UK

\section{G. Rayman}

The Ipswich Diabetes Centre and Research Unit, Ipswich Hospital NHS Trust, Colchester, Essex, UK

S. de Lusignan

Nuffield Department of Primary Care Health

Sciences, University of Oxford, Oxford, UK

M. Whyte

Clinical and Experimental Medicine, University of Surrey, Guildford, UK 
$2019,26.6 \%$ of deaths were of people with diabetes. Of this $26.6 \%, 18.5 \%$ would be expected from age group and non-diabetes status, while the other $8.1 \%$ would not have been expectedpro rata to nation, this approximates to approximately 40,000 excess deaths in people with diabetes vs the general population.

Conclusion: There remains a wide variation in mortality rate of people with diabetes between general practices in UK. The mortality rate and life years lost for people with diabetes vs nondiabetes individuals have remained stable in recent years, while mortality rates for the general population have fallen. Investment in diabetes management at a local and national level is enabling us to hold the ground regarding the life-shortening consequences of having diabetes as increasing numbers of people develop T2DM at a younger age.

Keywords: Diabetes; Mortality; Royal College of General Practitioners; General practice; Trend

\section{Key Summary Points}

Total population mortality rates have been falling and life expectancy increasing for more than 30 years. Diabetes remains a significant risk factor for premature death.

We used the Oxford Royal College of General Practitioners Research and Surveillance Centre (RCGP RSC) practices to determine diabetes-related vs nondiabetes-related mortality rates.

In 527 GP practices, we found no significant change in life years lost over the period 2004-2019 for diabetes individuals. This varied between 4.6 and 5.1 years over the period.

Investment at a local/national level in diabetes management in England may be enabling us to hold the ground regarding the life-shortening consequences of having diabetes as increasing numbers of people develop type 2 diabetes mellitus at a younger age.

\section{INTRODUCTION}

With sustained growth in diabetes numbers predicted for the future, effective early diagnosis and treatment of this condition along with sustained patient engagement are vital to avoid long-term comorbidities [1-3]. As new treatments are added to our current therapeutic armoury, there are significant opportunities for intervening early to improve the longer-term health outcomes for people with diabetes. The accumulation of comorbidities directly impacts on mortality, as does the quality of blood glucose control as measured by glycated haemoglobin A1c (HbA1c).

Using nationally available data, we previously showed that the higher mortality associated with a diagnosis of type 1 diabetes (T1DM) or type 2 diabetes (T2DM) could result in a loss of 6.4 million future life years in the current UK population [4]. In the model, the "average" person with T1DM (age 42.8 years) had a life expectancy from that age of 32.6 years, compared to 40.2 years for the equivalent-age nonDM individual-corresponding to 7.6 years of life years lost. The "average" person with T2DM (age 65.4 years) had a life expectancy from that age of 18.6 years, compared to 20.3 years for the equivalent-age individual without DM, corresponding to 1.7 life years lost per average person.

In 2017, Cavero-Redondo et al. [5] reported the results of a meta-analysis examining the relation between HbA1c level and all-cause mortality in people with diabetes. There was an increase in the risk of all-cause mortality when HbA1c levels were over $8.0 \%(64 \mathrm{mmol} / \mathrm{mol})$ or below $6 \%$ ( $42 \mathrm{mmol} / \mathrm{mol})$. The highest all-cause mortality in people with diabetes was an HbA1c above $9.0 \%$ (75 mmol/mol) (HR 1.69, 95\% CI 1.09-2.66). HbA1c levels above $7.0 \%$ $(53 \mathrm{mmol} / \mathrm{mol})$ were also associated with an increased risk of cardiovascular mortality in people with diabetes (HR 1.85, 95\% CI 1.14-2.55). This study suggested that people with diabetes should maintain their $\mathrm{HbA1c}$ in the range from $6.0 \%(42 \mathrm{mmol} / \mathrm{mol})$ to $7.0 \%$ $(53 \mathrm{mmol} / \mathrm{mol})$. 
A key question, therefore, is how all the developments in diabetes management have influenced mortality rates in people with diabetes over recent years. In this study, we evaluated-across all the general practices in the Oxford Royal College of General Practitioners Research and Surveillance Centre (RCGP RSC) the change in mortality over the years 2004-2019, and evaluated the variation in agestandardised mortality between general practices in the RCGP RSC.

\section{METHODS}

We used data from the RCGP RSC database [6]. This comprises primary care data from a sentinel network of primary care practices (general practices) distributed across England. Data were provided on patient numbers and deaths at practice level for those with diabetes, as well as for all adults, across four age groups $(<50$, $50-64,65-79, \geq 80$ years old). The model applied relative National Diabetes Audit (NDA) mortality rates to population rates for each age category and gender, and calculated the future life expectancy of individuals with diabetes (all types of diabetes) compared with non-DM populations for each general practice for the period 2004-2019. Matching the difference in total life expectancy for the reported populations by age and gender of the diabetes individuals to an equivalent population with nonDM gave the lost life years.

In order to demonstrate the generalisability of our findings, we also examined how representative the RCGP RSC data are in relation to all general practices in England. An additional "virtual" clinical commissioning group (CCG) that consisted of the RCGP RSC practices was created using publicly published data, and the results for this virtual RCGP-CCG were compared to those for other real CCGs. The measures included 35 indicators covering demography, services, medication, costs and outcomes. The ranking of the RCGP-CCG was then examined to see the positioning of these indicators compared to those for other CCGs.

\section{Data Analysis}

Annual data for each GP practice were taken from private and public sources, including:

RCGP RSC:

- List of participating practices

- Anonymous practices with the practice population categorised into four age groups $(<50,50-64,65-79, \geq 80$ years old) for 2004-2019

- Annual numbers for total population and deaths

- Annual numbers for total people with diabetes and deaths

National Diabetes Audit (performed annually during 2013-2019 for each participating practice):

- Total numbers with T1DM and T2DM split by age group $(<50,50-64,65-79, \geq 80$ years old), deprivation, ethnicity, sex

- Numbers with T1DM and T2DM completing service checks

- Numbers with T1DM and T2DM, with $\mathrm{HbA1c}$ results in ranges

GP practice prescribing data:

- By practice and BNF code

- Monthly amount (items, costs, quantity)

- Total amount

GP practice demographics 2013-2019:

- Total numbers in April of each year

- Age and gender split

Office for National Statistics (ONS)England:

- Life tables: life expectancy by age and sex (2004-2019)

- Death and population by age and sex (2004-2019). 
The mortality rate for the non-diabetes population was calculated as the difference between the totals minus those with diabetes. Expected deaths with diabetes was calculated by multiplying the annual mortality rate for the non-diabetes population in each age group by the equivalent diabetes population and then summing the results obtained for all age groups. Life expectancy years lost was calculated by applying the expected average life expectancy in each age group to the actual deaths and expected deaths of those with diabetes. Dividing the excess life expectancy years lost by the total number of deaths of those with diabetes gave the excess life expectancy loss for each diabetes death.

Multivariate analysis of variance (Manova) was used to compare trends in prescribing over the years 2014-2019 (IBM SPSS Statistics for Windows, version 24.0, Armonk, NY, USA).

\section{Ethics}

This project used aggregated general practice level data only and was categorised as a clinical audit. Permissions were received from RCGP RSC for data access. The study was performed in accordance with the Helsinki Declaration of 1964 and its later amendments.

\section{RESULTS}

We included 527 out of 550 general practices that were able to provide data covering the years 2004-2019 inclusive and participated in the National Diabetes Audit (NDA). There was no significant difference in key indicators between the RCGP RSC general practices $(n=550)$ and the non-RCGP-RCS practices in England $(n=6163)$ (Fig. 1$)$.

Table 1 shows a summary of the numbers of people and deaths in the total population and in those with diabetes for the years 2004-2019 recorded by the RCGP practices.

Over the period 2004-2019, there was no significant change in excess life years lost, which varied between 4.6 and 5.1 years per diabetes death. Table 2 summarises the increase in spend on medication for people with diabetes

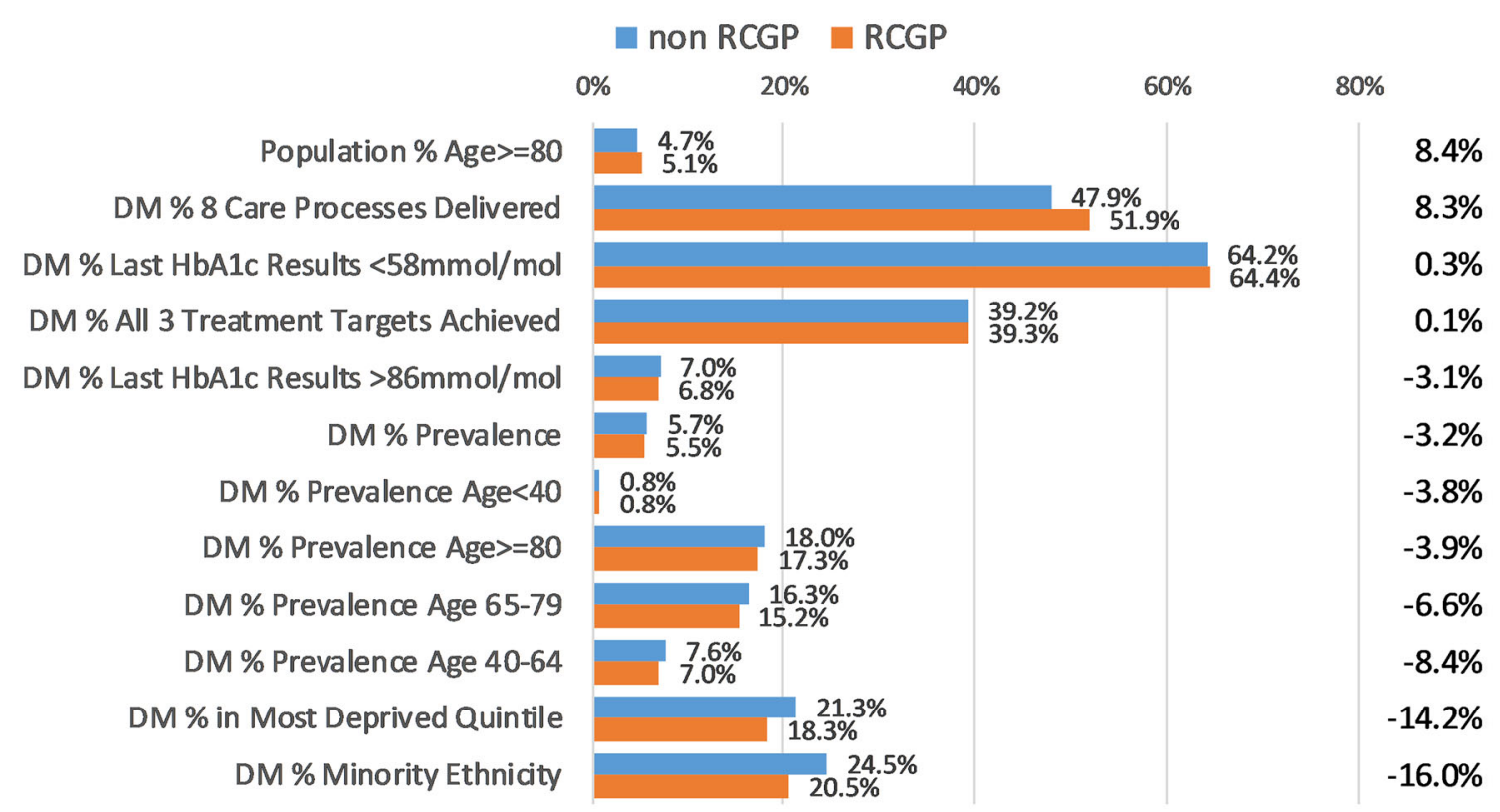

Fig. 1 Comparison between the average for all 550 RCGP practices and the average for the 6163 other practices in England in terms of key performance indicators Data taken from 2019-2020 National Diabetes Audit. The right-hand \% shows the difference, and the differences are ordered from high to low 


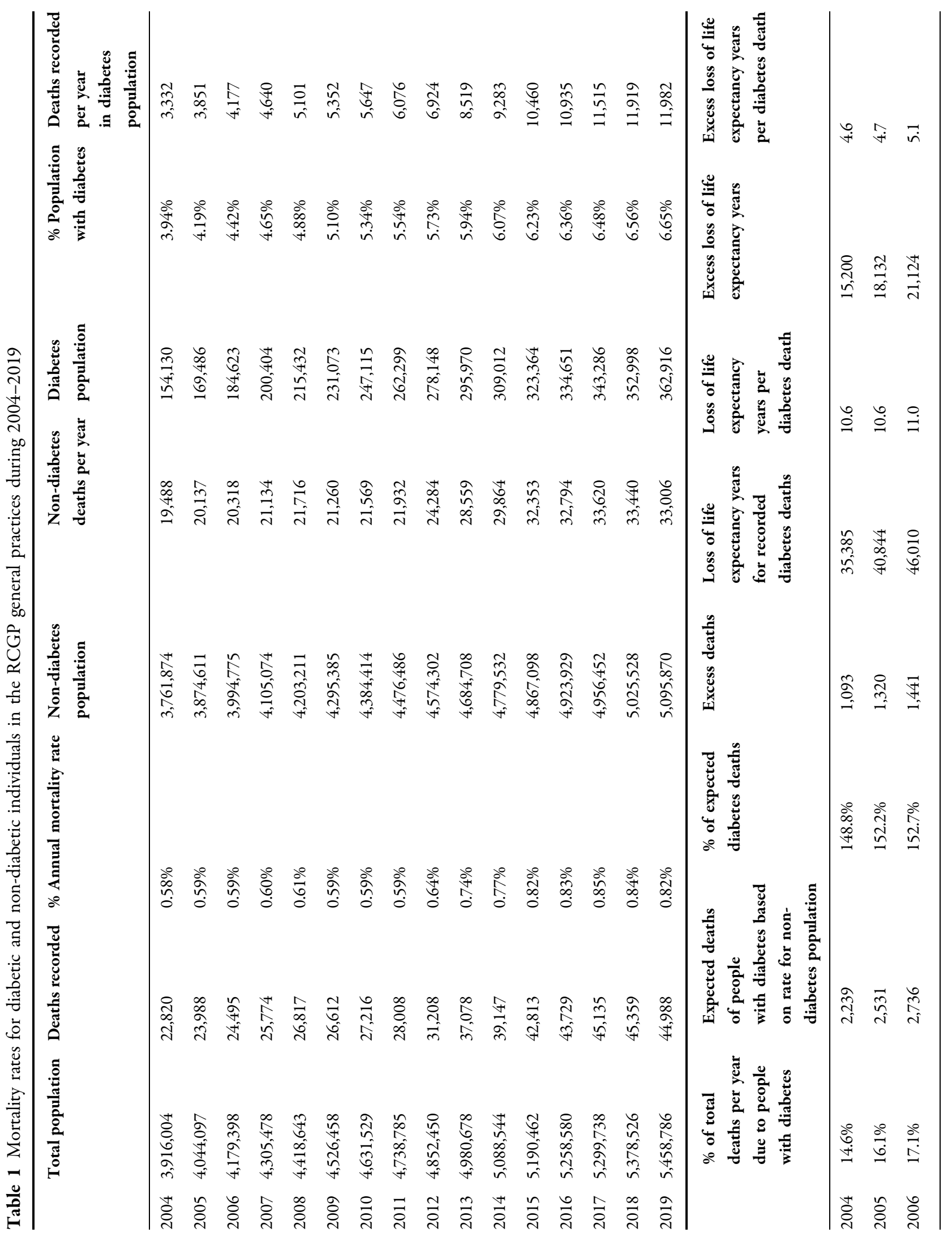




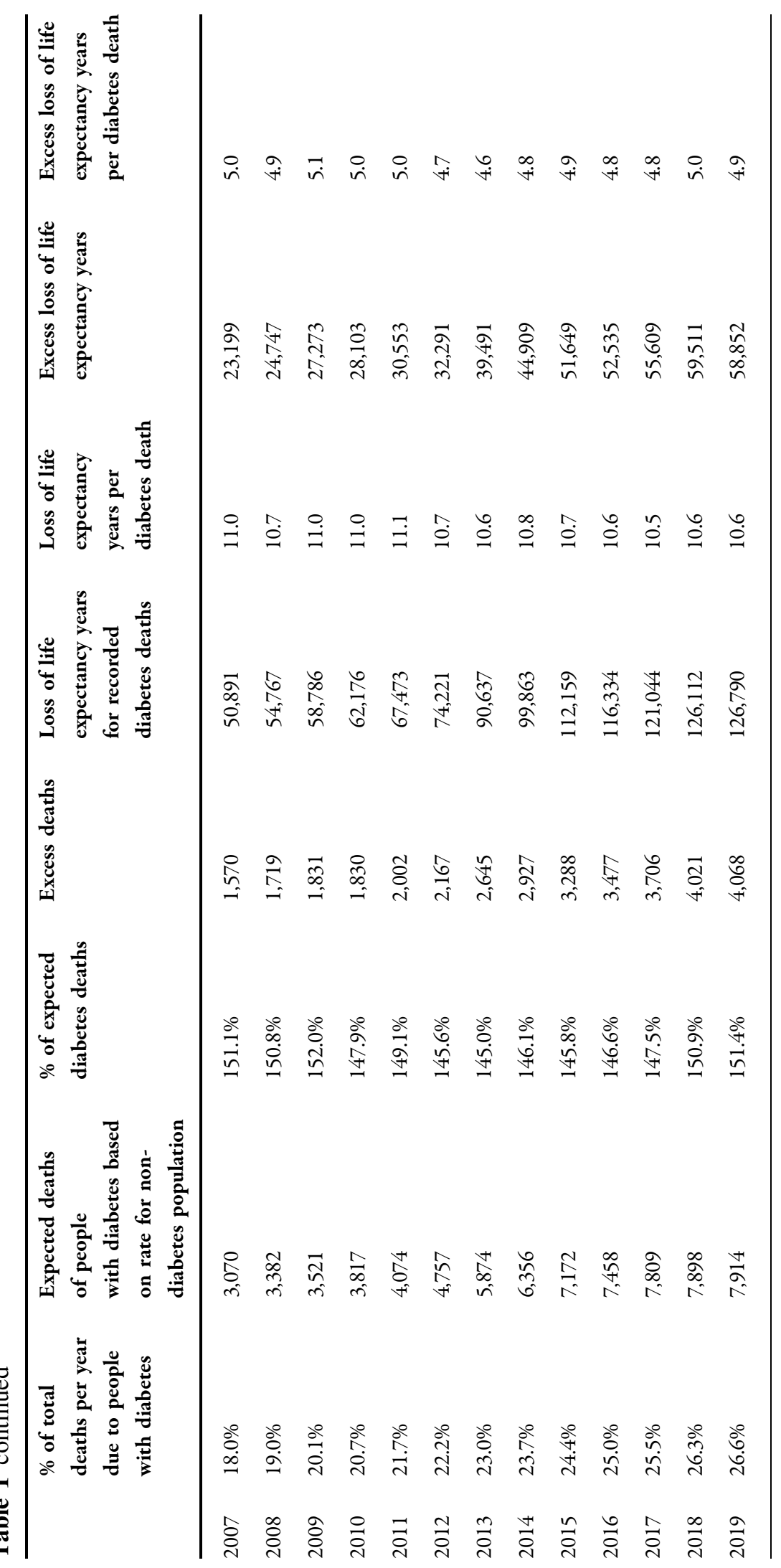


Table 2 Year-on-year spend on diabetes prescribing during 2014-2019

\begin{tabular}{|c|c|c|c|c|c|c|}
\hline & 2014 & 2015 & 2016 & 2017 & 2018 & 2019 \\
\hline Diabetes patients & 307,355 & 322,039 & 333,368 & 342,119 & 351,780 & 361,676 \\
\hline Diabetes medication prescriptions (millions) & 3.82 & 4.01 & 4.26 & 4.47 & 4.73 & 5.01 \\
\hline Diabetes medication cost $(£ \mathrm{~m})$ & $£ 65$ & $£ 71$ & $£ 76$ & $£ 80$ & $£ 87$ & $£ 94$ \\
\hline Cost/patient/year & $£ 211.93$ & $£ 221.96$ & $£ 229.17$ & $£ 232.79$ & $£ 246.62$ & $£ 260.02$ \\
\hline Cost/prescription & $£ 17.04$ & $£ 17.81$ & $£ 17.92$ & $£ 17.83$ & $£ 18.35$ & $£ 18.76$ \\
\hline
\end{tabular}

who attended RCGP RSC practices between 2014 and 2019 on a total and a per-patient basis.

For the year $2019,26.6 \%$ of the total deaths were of people with diabetes. Of that $26.6 \%$, $18.5 \%$ could be expected due to the ages of those people with diabetes. However, the additional $8.1 \%$ was an excess related to people who had diabetes while being treated. Pro rata to the national level for England, this approximates to 40,000 excess deaths annually within the treated diabetes population.

Figures $2 \mathrm{a}$ shows the distribution of deaths for the year 2019 between non-diabetes and diabetes individuals in the RCGP RSC. Specifically, the first and third horizontal bars in Fig. 2a show the age distributions of the nondiabetes and diabetes populations, respectively,
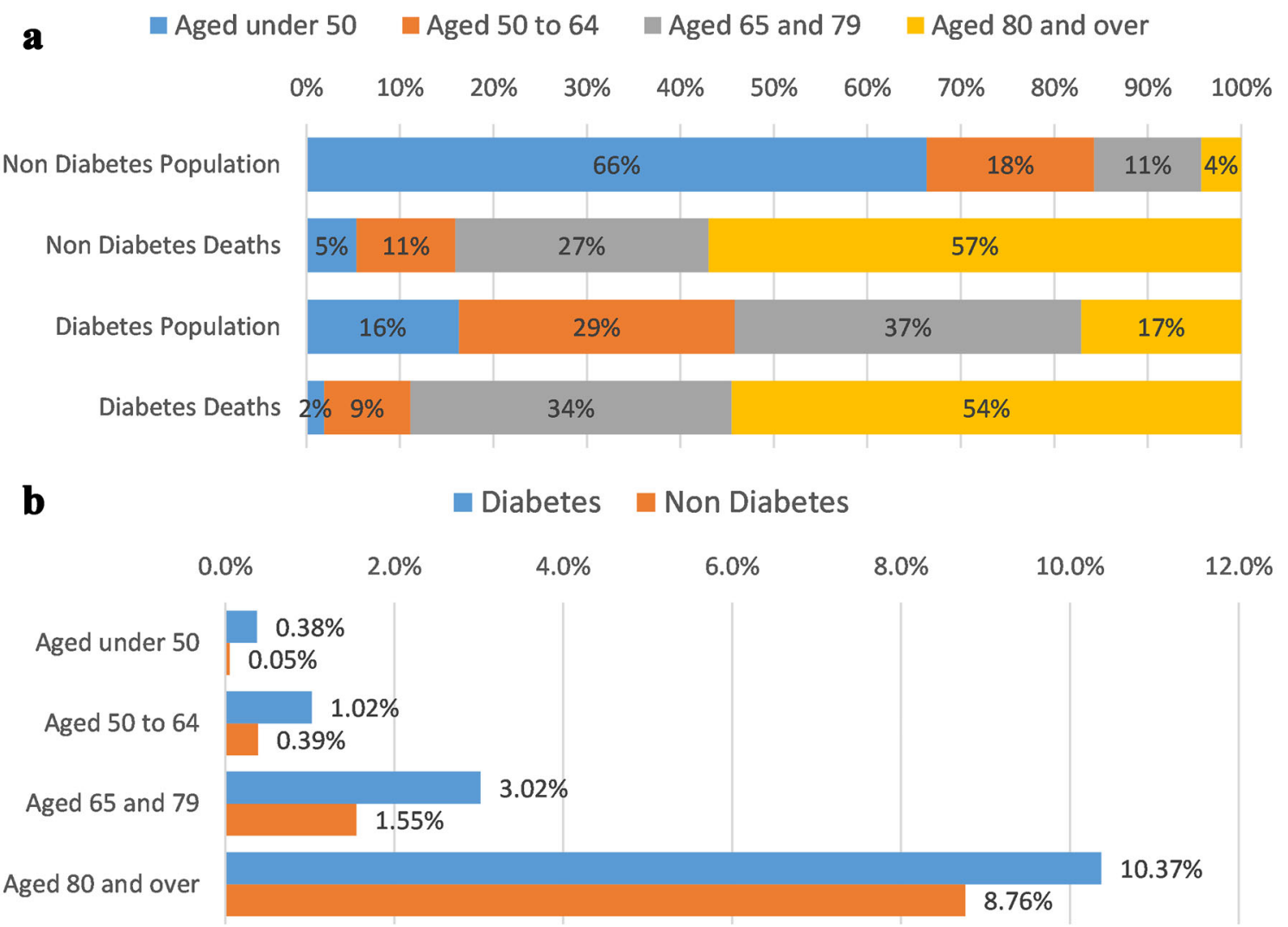

Fig. 2 a Distribution of deaths between non-diabetes and diabetes individuals in the RCGP practices for 2019. b Annualised mortality rates for non-diabetes and diabetes individuals in the RCGP practices for 2019 


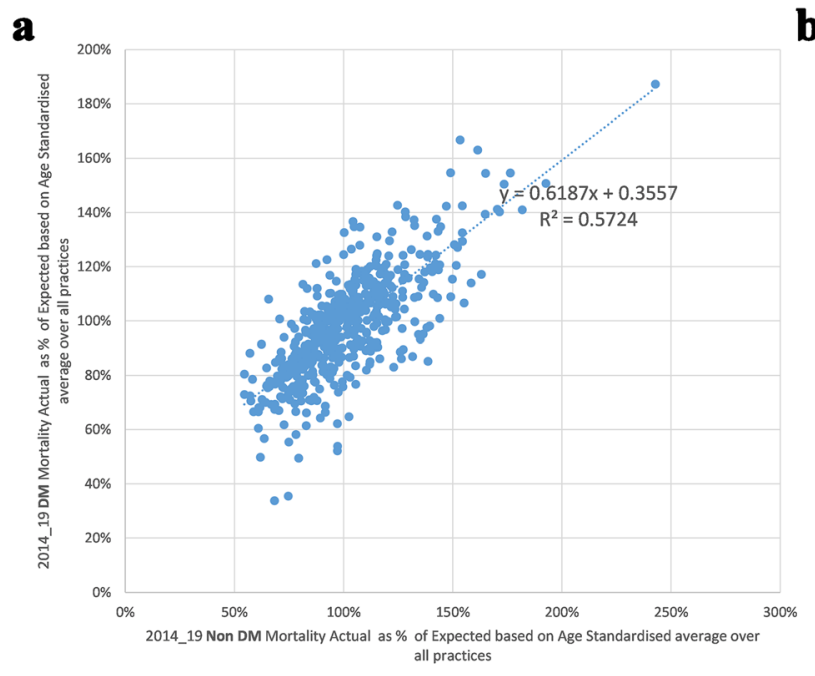

Fig. 3 a Relative deaths standardised by age in the average diabetes and non-diabetes populations during 2014-2019 for all the RCGP practices included in the study. $\mathbf{b}$ The practice-level variation in mortality rate for people with b

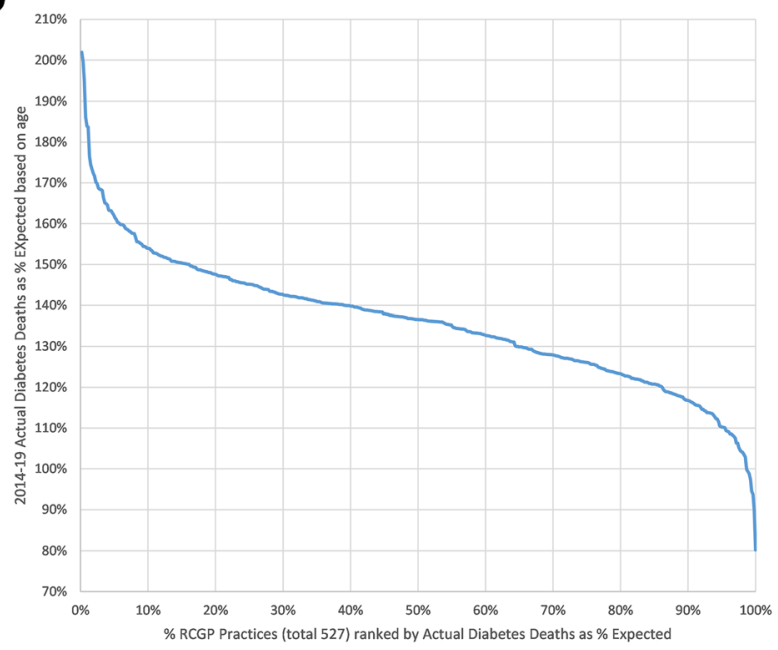

diabetes collated for the years 2014-2019. The RCGP practices are ranked by decile of actual diabetes deaths as a percentage of expected deaths

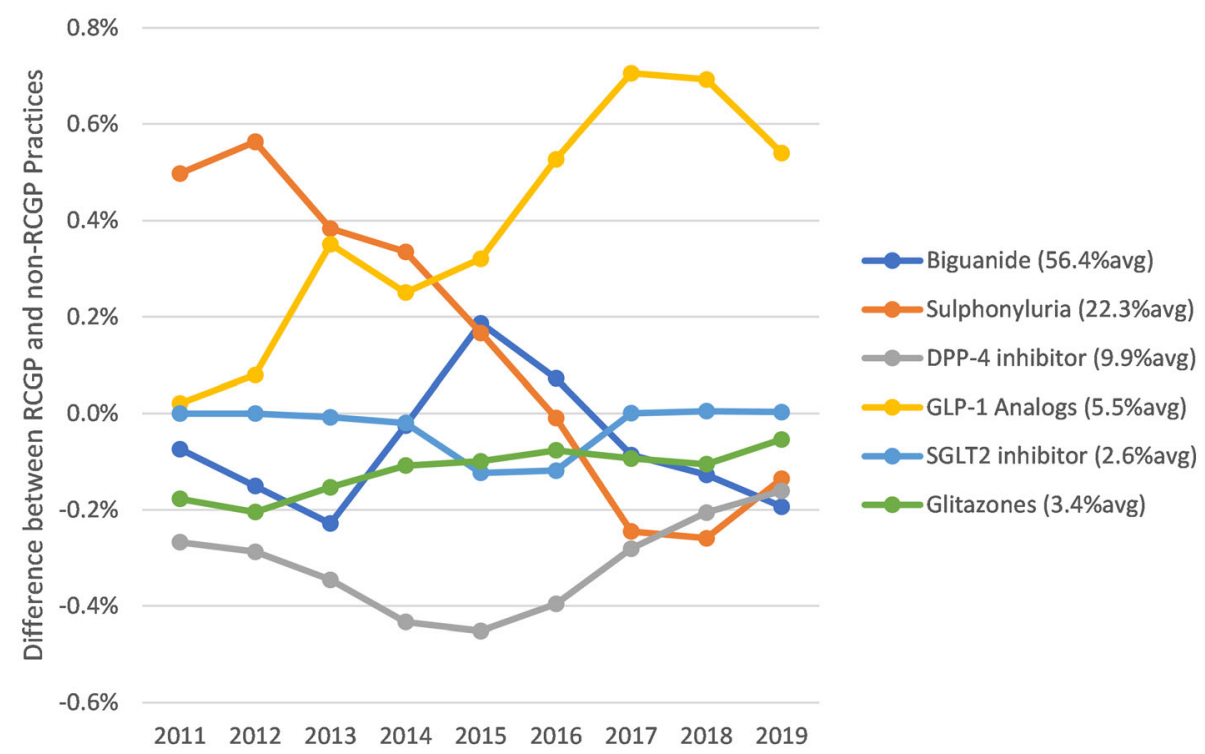

Fig. 4 Annual daily quantity (ADQ) comparison between classes of agent for RCGP vs non-RCGP practices in England during 2011-2019. The figure shows the

and the second and fourth horizontal bars show the proportion of deaths in each age band for the non-diabetes and diabetes populations. Figure $2 \mathrm{a}$ shows that the proportion of all comparative difference over time between RCGP and non-RCGP practices in relation to prescribing for this period

diabetes deaths by age band is higher for diabetes men and women in the 65-79 years age group than for their non-diabetic counterparts. Figure $2 b$ describes the annualised mortality 
rate for 2019 between non-diabetes and diabetes individuals in the RCGP RSC. We found that the difference in mortality rate between diabetes and non-diabetes individuals was much greater for younger individuals than for older individuals.

In Fig. 3a, we see the relation between the actual and expected deaths for non-diabetes and diabetes individuals at the general practice level for all RCGP RSC practices during the period 2014-2019. We defined the expected rate of diabetes death as the death rate based on non-diabetes deaths. The relative likelihood of death for diabetes vs non-diabetes people runs in relative synchrony, although there is practice-level variation such that there is a marked difference between the mortality rates of diabetes and non-diabetes individuals for some practices. We found that there was variation among practices in the actual vs expected deaths for the same period, as shown in Fig. 3b. For $68 \%$ of the general practices, the excess diabetes deaths were between 30 and $70 \%$ of the non-diabetes deaths, but some other practices had higher or lower excess deaths.

There was a significant difference between the RCGP RSC and non-RCCP practices in prescribing trends over the period 2014-2019, with a trend towards a greater relative use of glucagon-like peptide-1 (GLP-1) agonists ( $F=4.0$, $p=0.001)$ and a relative decrease in the use of sulphonylurea (SU) drugs $(F=4.2, p<0.001)$ (Fig. 4). For the RCGP RSC practices, overall annual spend on diabetes prescribing increased year on year, as shown in Table 2 .

\section{DISCUSSION}

We used the RCGP RSC data for this analysis. The fact that the RCGP general practices fall so close to the CCG median for each indicator (Fig. 1) shows that the RCGP network can be used as a representative group for studying practice-level diabetes performance.

In the context of advances in both the lifestyle of diabetes patients and the pharmacological management of diabetes as well as the increasing spend on diabetes prescribing (as shown for 2014-2019 in Table 2), we report that the mortality rate for people with diabetes has remained stable over the last 15 years. Conversely, the mortality rate in the general population has decreased, albeit more slowly in the last decade [7]. This lack of synergy in mortality rate with the general population may relate to people developing T2DM at an earlier age than in previous decades, mirroring the rising prevalence of obesity in younger-aged people $[8,9]$, with consequences for longer-term health outcomes, including mortality. We studied population-level effects and did not look in precise detail at specific individual factors and their changes over time. Many of these factors affect the non-diabetes population as well as people with diabetes, and have therefore been accounted for in the standardisation process.

Of relevance, the difference in annualised mortality rate was most pronounced for younger individuals (Fig. 2b). There was considerable variation between the diabetes-related excess deaths across general practices, as shown in Fig. 3b, where general practices are ranked by decile of actual diabetes deaths as a percentage of expected deaths. While some of this variation likely relates to underlying differences in demographic profile in terms of levels of social disadvantage and ethnic variation, our previous work [10-12] would suggest that modifiable factors such as general practice configuration/ CCG organisation of services and prescribing habits may also play a part. The relative reduction in sulphonylurea prescribing and relative increase in GLP-1 prescribing in the RCGP RSC practices vs other practices in England is a largescale example of such a phenomenon.

While we were not able to undertake an analysis using HbA1c values per se, the majority of people with diabetes will, by definition, have higher HbA1c levels than those of people without diabetes. Most population-based studies have found a J- or U-shaped association between HbA1c and mortality [13-19], with an optimal range from $5.6 \%(38 \mathrm{mmol} / \mathrm{mol})$ to $7.5 \%(58 \mathrm{mmol} / \mathrm{mol})[14,16]$, close to that in the report of Cavero-Redondo et al. [5]. Some studies have also reported a non-significant increase in all-cause mortality risk with each $0.1 \%$ increase in HbA1c (HR 1.14, 95\% CI 0.98-1.33) [20]. 
The association between HbA1c level and mortality is affected by age, diabetes duration and complications [21, 22]. In older diabetes patients, a high $\mathrm{HbA1c}$ (> 8\% or $64 \mathrm{mmol} / \mathrm{mol}$ ) level was associated with an increased risk of allcause mortality, while there is less evidence about the relation between low/normal HbA1c level and mortality $[15,16,22]$. In people with a relatively short diabetes duration ( $<5$ years), the effect of a low normal HbA1c level on subsequent all-cause mortality remains unclear $[13,17,20,21]$; on the other hand, for people with a relatively long duration of disease ( $\geq 5$ years), a low HbA1c level was associated with a higher risk of mortality during subsequent follow-up [21].

In the study by Raghavan et al. of people with T2DM and coronary artery disease, only a lower level of HbA1c (below 6\%; $42 \mathrm{mmol} / \mathrm{mol}$ ) was associated with a higher risk of 1-year mortality; a higher level of HbA1c was not associated with mortality [23]. Thus, there remains a need to explore the relation between HbA1c level and mortality in diabetes people with different characteristics via larger population studies that factor duration of elevation of HbA1c into the longitudinal analysis.

Finally, in our previous analysis [4], as described in the "Introduction", the life years lost was slightly different when T1DM and T2DM outcomes were analysed separately. This will, at least in part, relate to a different standardised mortality ratio for diabetes in this analysis (1.50) compared to the 2014/15 NDA standardised mortality ratio (1.33). It should also be pointed out that, in spite of the retrospective nature of the analysis and the variability in data collection approaches across several hundred general practices, we have been able to draw conclusions that are relevant to the health and life prospects of people with diabetes in the twenty-first century.

\section{Strengths/Limitations}

We were not able to differentiate T1DM and T2DM from the practice-level data. Approximately $90 \%$ of diabetes is T2DM, so this has the greatest effect on the data, which nevertheless cover all types of diabetes.

Furthermore, we know that there are individuals with no symptoms of T2DM but high blood glucose levels who have not been diagnosed because they have not had the relevant blood test. They would thus have been categorised as not having diabetes.

This study was an audit evaluation and therefore permissions were not sought for linkage identification of general practices. Hence, we did not link with any practice-level datasets beyond the RCGP RSC.

Any registry-based study of this kind will have inherent biases. However, we have confirmed that the RCGP RSC practices [24] are representative of the practices in the rest of England. Finally, we did not include data on mediators of health outcome such as HbA1c, renal indices, blood pressure, ethnicity and socioeconomic status. These data will form part of further analyses that are ongoing.

We have no data on smoking and alcohol consumption. However, there is no reason to suspect that smoking and alcohol consumption would be different in diabetes vs non-diabetes individuals.

\section{CONCLUSIONS}

There remains a wide variation in mortality rate in people with diabetes between general practices. The mortality rate and life years lost for people with diabetes vs non-diabetes individuals have remained reasonably stable in recent years, while the mortality rate for the general population has fallen. The factors underlying this phenomenon remain to be characterised. Investment at a local and national level in diabetes management is enabling us to hold the ground regarding the life-shortening consequences of having diabetes as increasing numbers of people develop T2DM at a younger age. 


\section{ACKNOWLEDGEMENTS}

Funding. There was no specific funding for this study. The Rapid Service Fee was funded by the authors.

Authorship. All named authors meet the International Committee of Medical Journal Editors (ICMJE) criteria for authorship for this article, take responsibility for the integrity of the work as a whole, and have given their approval for this version to be published.

Author Contributions. Adrian Heald, Mike Stedman, Adam Robinson, Mark Davies, Mark Livingston, Ramadan Alshames, Gabriela Moreno, Roger Gadsby, Gerry Rayman, Martin Gibson, Simon de Lusignan and Martin Whyte contributed equally to the conception, design, data analysis and writing up of this paper.

Disclosures. Adrian Heald, Mike Stedman, Adam Robinson, Mark Davies, Mark Livingston, Ramadan Alshames, Gabriela Moreno, Roger Gadsby, Gerry Rayman, Martin Gibson, Simon de Lusignan and Martin Whyte do not have any conflict of interest.

Compliance with Ethics Guidelines. This project used aggregated general practice level data only and was categorized as a Clinical Audit. Permissions were received from RCGP RSC for data access.

Data Availability. The datasets generated during and/or analyzed during the current study are not publicly available as all data access is under the jurisdiction of the RCGP RSC Governance structure.

Open Access. This article is licensed under a Creative Commons Attribution-NonCommercial 4.0 International License, which permits any non-commercial use, sharing, adaptation, distribution and reproduction in any medium or format, as long as you give appropriate credit to the original author(s) and the source, provide a link to the Creative Commons licence, and indicate if changes were made. The images or other third party material in this article are included in the article's Creative Commons licence, unless indicated otherwise in a credit line to the material. If material is not included in the article's Creative Commons licence and your intended use is not permitted by statutory regulation or exceeds the permitted use, you will need to obtain permission directly from the copyright holder. To view a copy of this licence, visit http://creativecommons.org/licenses/by$\mathrm{nc} / 4.0 /$.

\section{REFERENCES}

1. Fisher L, Polonsky WH, Hessler D, Potter MB. A practical framework for encouraging and supporting positive behaviour change in diabetes. Diabet Med. 2017;43(12):1658-66.

2. The DCCT/EDIC Research Group. Epidemiology of Diabetes Interventions and Complications (EDIC). Design, implementation, and preliminary results of a long-term follow-up of the Diabetes Control and Complications Trial cohort. Diabetes Care 1999;22:99-111.

3. Alva ML, Gray A, Mihaylova B, Leal J, Holman RR. The impact of diabetes-related complications on healthcare costs: new results from the UKPDS (UKPDS 84). Diabet Med. 2015;32:459-66.

4. Heald AH, Stedman M, Davies M, Livingston M, Alshames R, Lunt M, Rayman G, Gadsby R. Estimating life years lost to diabetes: outcomes from analysis of National Diabetes Audit and Office of National Statistics data. Cardiovasc Endocrinol Metab. 2020;9:183-5.

5. Cavero-Redondo I, Peleteiro B, Alvarez-Bueno C, Rodriguez-Artalejo F, Martinez-Vizcaino V. Glycated haemoglobin A1c as a risk factor of cardiovascular outcomes and all-cause mortality in diabetic and non-diabetic populations: a systematic review and meta-analysis. BMJ Open. 2017;7: e015949.

6. Oxford Royal College of General Practitioners (RCGP) Research and Surveillance Centre. Public health data. https://www.rcgp.org.uk/clinical-andresearch/our-programmes/research-andsurveillance-centre/public-health-data.aspx. Accessed 12 May 2021

7. Appleby J. UK deaths in 2020: how do they compare with previous years? BMJ 2021;373:n896. 
8. Public Heath England. Adult obesity and type 2 diabetes. https://assets.publishing.service.gov.uk/ government/uploads/system/uploads/attachment_ data/file/338934/Adult_obesity_and_type_2_diabetes_. pdf. Accessed 1 August 2021.

9. Schienkiewitz A, Schulze MB, Hoffmann K, Kroke A, Boeing H. Body mass index history and risk of type 2 diabetes: results from the European Prospective Investigation into Cancer and Nutrition (EPIC)Potsdam Study. Am J Clin Nutr. 2006;84:427-33.

10. Heald $\mathrm{AH}$, Livingston $\mathrm{M}$, Malipatil N, Becher $\mathrm{M}$, Craig J, Stedman M, Fryer AA. Improving type 2 diabetes mellitus glycaemic outcomes is possible without spending more on medication: Lessons from the UK National Diabetes Audit. Diabetes Obes Metab. 2018;20:185-94.

11. Heald AH, Livingston $M$, Fryer A, Moreno GYC, Malipatil N, Gadsby R, Ollier W, Lunt M, Stedman M, Young RJ. Route to improving Type 1 diabetes mellitus glycaemic outcomes: real-world evidence taken from the National Diabetes Audit. Diabet Med. 2018;35:63-71.

12. Stedman $M$, Lunt $M$, Davies $M$, Livingston $M$, Duff C, Fryer A, Anderson SG, Gadsby R, Gibson M, Rayman G, Heald A. Cost of hospital treatment of type 1 diabetes (T1DM) and type 2 diabetes (T2DM) compared to the non-diabetes population: a detailed economic evaluation. BMJ Open. 2020;10: e03323.

13. Li FR, Zhang XR, Zhong WF, Li ZH, Gao X, Kraus $\mathrm{VB}$, et al. Glycated hemoglobin and all-cause and cause-specific mortality among adults with and without diabetes. J Clin Endocrinol Metab. 2019;104:3345-54.

14. Raghavan S, Vassy JL, Ho YL, Song RJ, Gagnon DR, Cho K, et al. Diabetes mellitus-related all-cause and cardiovascular mortality in a national cohort of adults. J Am Heart Assoc. 2019;8: e011295.

15. Hamada S, Gulliford MC. Mortality in individuals aged 80 and older with type 2 diabetes mellitus in relation to glycosylated hemoglobin, blood pressure, and total cholesterol. J Am Geriatr Soc. 2016;64:1425-31.
16. Doucet J, Verny C, Balkau B, Scheen AJ, Bauduceau B. Haemoglobin A1c and 5-year all-cause mortality in French type 2 diabetic patients aged 70 years and older: The GERODIAB observational cohort. Diabetes Metab. 2018;44:465-72.

17. Navarro-Perez J, Orozco-Beltran D, Gil-Guillen V, Pallares V, Valls F, Fernandez A, et al. Mortality and cardiovascular disease burden of uncontrolled diabetes in a registry-based cohort: the ESCARVAL-risk study. BMC Cardiovasc Disord. 2018;18:180.

18. Reges O, Leibowitz M, Hoshen M, Leventer-Roberts M, Greenland P, Balicer R. Diabetes control: Incidence of acute myocardial infarction and all-cause mortality among patients with 3-6 years' disease duration. Eur J Prev Cardiol. 2017;24:1083-92.

19. Li W, Katzmarzyk PT, Horswell R, Wang Y, Johnson J, Hu G. HbA1c and all-cause mortality risk among patients with type 2 diabetes. Int $\mathrm{J}$ Cardiol. 2016;202:490-6.

20. Rozing MP, Moller A, Aabenhus R, Siersma V, Rasmussen K, Koster-Rasmussen R. Changes in HbA1c during the first six years after the diagnosis of Type 2 diabetes mellitus predict long-term microvascular outcomes. PLoS One. 2019;14: e0225230.

21. Davis TM, Chubb SA, Bruce DG, Davis WA. Metabolic memory and all-cause death in communitybased patients with type 2 diabetes: the Fremantle Diabetes Study. Diabetes Obes Metab. 2016;18: 598-606.

22. Palta P, Huang ES, Kalyani RR, Golden SH, Yeh HC. Hemoglobin A1c and mortality in older adults with and without diabetes: results from the National Health and Nutrition Examination Surveys (1988-2011). Diabetes Care. 2017;40:453-60.

23. Raghavan S, Liu WG, Michael Ho P, Plomondon $\mathrm{ME}$, Baron AE, Caplan L, et al. Coronary artery disease severity modifies associations between glycemic control and both mortality and myocardial infarction. J Diabetes Compl. 2018;32:480-7.

24. Correa A, Hinton W, McGovern A, van Vlymen J, Yonova I, Jones S, de Lusignan S. Royal College of General Practitioners Research and Surveillance Centre (RCGP RSC) sentinel network: a cohort profile. BMJ Open. 2016;6(4): e011092. 